\title{
SEGURANÇA ALIMENTAR E ORGANIZAÇÃO MUNDIAL DO COMÉRCIO: ANÁLISE CRÍTICA DO ACORDO SOBRE A APLICAÇÃO DE MEDIDAS SANITÁRIAS E FITOSSANITÁRIAS
}

\author{
FOOD SAFETY AND WORLD TRADE ORGANIZATION: CRITICAL \\ ANALYSIS OF THE AGREEMENT ON THE APPLICATION OF SANITARY \\ AND PHYTOSANITARY MEASURES
}

\author{
Eugênia Cristina Nilsen Ribeiro Barza* \\ Natália Paulino Bonnomi**
}

\begin{abstract}
Resumo: O objeto deste artigo é a análise do impacto do Acordo sobre a Aplicação de Medidas Sanitárias e Fitossanitárias (Acordo SPS) nas políticas regulatórias direcionadas à proteção da segurança alimentar. A problematização do trabalho consiste em demonstrar como o Acordo SPS pode orientar a regulação do Estado para que esta alcance o nível desejado de proteção sem ensejar barreiras protecionistas ao comércio. Para tanto, o presente artigo estuda as principais normas contidas no Acordo SPS, bem como avalia a disputa EC-Biotech, de modo a compreender o posicionamento da OMC acerca da regulação relativa à segurança alimentar promovida pelos Estados. Conclui-se que são três os caminhos, que constituem direitos autônomos, para os Estados desenvolverem uma política regulatória de segurança alimentar em observância ao Acordo SPS: medidas que estão em conformidade com os padrões internacionais (Artigo 3.1); medidas, com justificação científica, que resultem em um nível mais elevado de proteção (Artigo 3.3); e, quando a evidência científica for insuficiente, medidas provisórias com base na informação pertinente disponível (Artigo 5.7).
\end{abstract}

Palavras-chave: Acordo SPS; comércio internacional; Organização Mundial do Comércio; regulação; segurança alimentar.

\begin{abstract}
The object of this paper is to analyze the impact of the Agreement on the Application of Sanitary and Phytosanitary Measures (SPS Agreement) in the regulatory policy directed to the protection of food safety. The problem faced in this paper is to demonstrate how the SPS Agreement can guide the regulation by the State so that it reaches the desired level of protection without placing protectionist barriers to trade. Therefore, this article examines the main rules contained in the SPS Agreement, as well as assesses the EC-Biotech dispute, in order to understand the WTO's perception of the regulation of food safety promoted by the States. It is concluded that there are three paths, which constitute autonomous rights, for States to develop a regulatory policy for food safety in compliance with the SPS Agreement: measures that comply with international standards (Article 3.1); measures, with scientific justification, that result in a higher level of protection (Article 3.3); and, when scientific evidence is insufficient, provisional measures based on relevant available information (Article 5.7).
\end{abstract}

\footnotetext{
* Professora Associada, Departamento de Direito Público Especializado, Faculdade de Direito do Recife/ Centro de Ciências Jurídicas, Universidade Federal de Pernambuco (UFPE) E-mail: ecnrbarza@terra.com.br.

** Advogada, Mestranda, Programa de Pós-Graduação em Direito, Universidade Federal de Pernambuco (UFPE). E-mail: nataliapbmi@gmail.com.
} 
Segurança alimentar e Organização Mundial do Comércio: análise crítica do acordo sobre a aplicação de medidas sanitárias e fitossanitárias

Keywords: Agreement SPS; international trade; food security; regulation; World Trade Organization.

\section{INTRODUÇÃO}

A segurança alimentar corresponde ao efetivo acesso da população a uma alimentação suficiente, nutritiva e segura. A condição de segurança dos alimentos demanda que estes não sejam prejudiciais à vida ou à saúde humana, animal ou vegetal. A preocupação dos Estados com a segurança alimentar gerou a proliferação de normas internas e internacionais - com o intuito de garantir a proteção das pessoas e do meio ambiente. No plano internacional, a temática da segurança alimentar ganhou importância especialmente a partir da elaboração do Codex Alimentarius, conjunto de normas relativas à proteção da saúde dos consumidores e às práticas justas no comércio de alimentos, estabelecido conjuntamente pela Organização das Nações Unidas para a Alimentação e a Agricultura (FAO) e pela Organização Mundial de Saúde (OMS), em 1963.

No entanto, os standards utilizados pelos Estados com o intuito de garantir a segurança alimentar de sua população podem acarretar grande impacto no comércio internacional, uma vez que a adoção de tais medidas pode gerar barreiras comerciais. Diante disso, foi criado o Acordo sobre a Aplicação de Medidas Sanitárias e Fitossanitárias (Acordo SPS), no âmbito normativo da Organização Mundial do Comércio (OMC), a fim de fixar os critérios em que as medidas são consideradas legítimas à proteção da segurança alimentar, isto é, quando não são empregadas como barreiras protecionistas ao comércio.

Sendo assim, o objetivo principal do presente artigo é demonstrar de que forma uma política estatal regulatória, visando à segurança alimentar, está de acordo com os preceitos de não discriminação defendidos pela OMC por meio do Acordo SPS. Este estudo também objetiva precisar os parâmetros definidos para legitimidade de uma medida voltada à proteção da segurança alimentar, através da análise do Acordo SPS, bem como da decisão emitida na controvérsia EC-Biotech pelo Órgão de Solução de Controvérsias (OSC). Por fim, pretende-se fazer uma avaliação crítica da interpretação conferida pela OMC ao Acordo SPS, de modo a determinar os possíveis caminhos disponíveis para os Estados na formação de sua política regulatória em conformidade com o Acordo SPS. 
A importância do trabalho reside na atualidade da discussão da segurança alimentar no comércio internacional, principalmente em virtude da elaboração do Acordo SPS. O artigo também se justifica em razão da relevância do posicionamento da OMC para a fixação das políticas regulatórias para proteger a saúde humana e o meio ambiente sem oferecer obstáculos protecionistas ao comércio.

O artigo será desenvolvido, em primeiro lugar, a partir da adoção do método bibliográfico, por meio da apresentação da doutrina sobre o tema. Além disso, a metodologia do trabalho consiste na análise dos dispositivos do Acordo SPS e da disputa EC-Biotech, submetida à OMC, a fim de se visualizar os critérios determinados pela Organização para uma legítima política de segurança alimentar, e como tal entendimento afeta os standards definidos pelos Estados.

A estrutura do artigo consiste na exposição de quatro capítulos. No primeiro, o trabalho irá tratar do conceito de segurança alimentar e de sua regulamentação no âmbito internacional. Posteriormente, no segundo capítulo, serão examinados os principais artigos do Acordo SPS, cujas normas tratam dos requisitos de legitimidade das medidas SPS. Após essa análise, no terceiro capítulo, será estudada a interpretação dos referidos dispositivos conferida pelo Órgão de Solução de Controvérsias (OSC) da OMC, assim como os pormenores da disputa EC-Biotech, cujo objeto foi a importação de organismos geneticamente modificados (OGMs). Finalmente, será abordada a relação entre os dispositivos do Acordo SPS e, consequentemente, de que formas os Estados podem construir sua política regulatória voltada à proteção da segurança alimentar em conformidade com o Acordo.

\section{SEGURANÇA ALIMENTAR E CODEX ALIMENTARIUS}

O conceito de segurança alimentar foi construído, originalmente, a partir da ideia de disponibilidade de alimentos considerada suficiente para cumprir os requisitos necessários para uma dieta de energia. Desse modo, o termo segurança alimentar costumava ser utilizado também para se referir à autossuficiência de um Estado, ou seja, de um país cuja produção de alimentos correspondia à necessidade ou à demanda de sua população. $\mathrm{O}$ conceito de segurança alimentar, portanto, esteve ligado à disponibilidade suficiente de alimentos para uma dieta de energia, o qual, contudo, não corresponde ao seu significado atual. A mera disponibilidade não assegura o acesso de toda a população 
Segurança alimentar e Organização Mundial do Comércio: análise crítica do acordo sobre a aplicação de medidas sanitárias e fitossanitárias

aos alimentos, bem como uma quantidade suficiente de calorias não garante uma dieta saudável e nutritiva (PINSTRUP-ANDERSEN, 2009, p. 5).

Sendo assim, o conceito de segurança alimentar é mais abrangente, e engloba elementos como o efetivo acesso das pessoas aos alimentos e uma alimentação nutritiva e segura. Tal acepção do termo segurança alimentar foi incorporada por estudiosos e profissionais, em meados da década de 1970, que a definiram como o acesso, por todas as pessoas, a uma alimentação suficiente para ter uma vida saudável e produtiva (PINSTRUP-ANDERSEN, 2009, p. 5-6).

Em 1991, a Conferência sobre Normas para Alimentos, Químicos em Alimentos e Comércio de Alimentos, realizada em Roma e organizada pela FAO e pela OMS, fortaleceu o Codex Alimentarius e comitês específicos para que continuassem a fornecer subsídios científicos para embasar as avaliações de risco. Em 1992, também em Roma, ocorreu a Conferência Internacional sobre Nutrição, a qual incentivou os governos a tomar as medidas necessárias para proteger a saúde e a segurança do consumidor por meio do suprimento de alimentos suficientes, de alta qualidade e seguros (RANDELL; WHITEHEAD, 1997, p. 313).

Nesse sentido, em 1996, foi realizada a Cúpula Mundial da Alimentação, por intermédio da FAO, na qual foram aprovados uma Declaração (Declaração de Roma sobre a Segurança Alimentar Mundial) e um Plano de Ação, com o objetivo de combater a fome no mundo. Nesses documentos, observa-se que a definição de segurança alimentar foi ampliada, e se diz que ocorre quando todas as pessoas têm, a todo momento, acesso físico e econômico a alimentos seguros, nutritivos e suficientes para satisfazer as suas necessidades dietéticas e preferências alimentares, a fim de levarem uma vida ativa e sã (PINSTRUP-ANDERSEN, 2009, p. 5-6).

O termo alimentos seguros e nutritivos destaca a segurança alimentar e a composição nutritiva da alimentação, enquanto a adição do termo preferências alimentares aprimora o conceito de segurança alimentar do simples acesso à alimentação suficiente para acesso à alimentação preferida. As preferências dizem respeito a alimentos aceitáveis social e culturalmente, bem como que respeitem valores de religião e ética (PINSTRUP-ANDERSEN, 2009; p. 5-6).

Dessa maneira, o aspecto da segurança alimentar que será tratado neste artigo diz respeito à condição de segurança dos alimentos disponíveis à população. Tal aspecto engloba, por exemplo, questões relativas a agrotóxicos, organismos geneticamente modificados, técnicas que afetam o meio ambiente, entre outras. Com a evolução do 
conceito de segurança alimentar, como abordado acima, e com a crescente preocupação com o consumo de alimentos seguros para a saúde humana e para o ambiente, os organismos internacionais e os Estados passaram a regulamentar e a exercer um maior controle sobre a entrada de gêneros alimentícios em seus territórios.

$\mathrm{Na}$ seara internacional, o instrumento que prevê padrões de alimentação, orientações e códigos de prática, aptos a proteger a saúde do consumidor e as práticas justas no comércio de alimentos, é o já citado Codex Alimentarius, estabelecido pela FAO e pela OMS em 1963. Os standards dispostos no Codex são baseados nas evidências científicas disponíveis mais adequadas, e tratam de diversas questões relacionadas à segurança alimentar, tais como padrões para determinados alimentos, rotulagem, higiene de alimentos, biotecnologia, comércio de alimentos, entre outros. Embora esses standards sejam de adesão voluntária pelos Membros, são bastante utilizados como base para legislações nacionais ${ }^{1}$.

Os padrões previstos no Codex Alimentarius pretendem assegurar um patamar mínimo de qualidade do alimento, bem como promover recomendações para boas práticas regulatórias. Um dos principais objetivos do Codex, ao criar um conjunto uniforme de standards, é proporcionar uma harmonização na regulação doméstica de forma a facilitar o comércio internacional (LIVERMORE,2006, p. 771).

O Codex Alimentarius, apesar de ter trazido importantes parâmetros no que diz respeito à adoção e à implementação de medidas direcionadas à qualidade dos alimentos e à segurança alimentar, não era de cumprimento obrigatório, o que apenas ocorria quando o Estado expressamente adotava seus standards. Dessa forma, os Estados estavam livres para decidir se adotariam ou não as normas do Codex e em que grau sua política de regulação seria compatível com elas (ETCHELAR; MALJEAN-DUBOIS, 2007, p. 140).

Entretanto, os padrões de segurança alimentar constantes no Codex servem de base para o Acordo SPS da OMC, o que significa que os seus standards são utilizados como indicadores para a formulação de políticas internas, bem como para solução de controvérsias relativas ao comércio entre Estados. Os Membros da OMC devem basear suas medidas concernentes à segurança alimentar nos standards, diretrizes $\mathrm{e}$ recomendações do Codex Alimentarius, visto que, uma vez que a medida esteja de acordo com o Codex, há uma presunção de sua consistência com o Acordo SPS. Sendo assim, as disposições do Codex Alimentarius, ainda que de cumprimento voluntário em princípio, 
Segurança alimentar e Organização Mundial do Comércio: análise crítica do acordo sobre a aplicação de medidas sanitárias e fitossanitárias

tornaram-se vinculantes na esfera do comércio internacional através do Acordo SPS (TOMA-BIANOV, 2013, p. 192).

Portanto, o Acordo SPS permitiu que padrões internacionais relativos à proteção da saúde e do meio ambiente, que antes eram trazidos apenas como recomendações não vinculantes, fossem mandatórios nas relações comerciais. Sendo assim, as medidas voltadas à segurança alimentar têm sido cada vez mais harmonizadas, conforme os ditames do Codex Alimentarius, em virtude do status de obrigatoriedade reservado às suas normas pelo Acordo SPS.

\section{SEGURANÇA ALIMENTAR E SEU IMPACTO NO COMÉRCIO INTERNACIONAL: ANÁLISE DO ACORDO SPS}

Pode-se observar que a segurança alimentar tem sido uma matéria de bastante relevância nas políticas regulatórias dos Estados. A partir de ações internacionais, promovidas principalmente pela FAO, a condição de segurança dos alimentos que circulam nos territórios dos países assumiu um papel fundamental no que tange à produção e à exportação de gêneros alimentícios. Os produtores e os exportadores de alimentos têm que adequar seus produtos aos padrões estabelecidos pelos Estados, os quais regulamentam diversas questões, tais como organismos geneticamente modificados, pesticidas, hormônios, entre outras.

Daí se percebe, então, que os standards regulatórios relativos à segurança alimentar podem gerar enormes impactos no comércio internacional. Dessa maneira, na Rodada Tóquio (1973-79) do Acordo Geral sobre Tarifas e Comércio (GATT), foi estabelecido o Standards Code (Código de Normas), de adesão voluntária, que objetivava disciplinar questões de regulamentos técnicos, inclusive medidas de natureza sanitária. As negociações sobre a matéria avançaram durante a Rodada Uruguai (1986-94), e o Standards Code foi sucedido por dois acordos, o Acordo sobre a Aplicação de Medidas Sanitárias e Fitossanitárias (Acordo SPS) e o Acordo sobre Barreiras Técnicas (Acordo TBT) (BARROS; BURNQUIST; CUNHA FILHO; MIRANDA, 2004, p. 26).

O Acordo SPS é destinado a regulamentar as medidas referentes à vida ou à saúde humana, animal ou vegetal que afetem o comércio internacional. Essas medidas englobam normas, métodos, procedimentos, exigências relacionadas diretamente à segurança alimentar. Já o Acordo TBT pretende garantir que os procedimentos concernentes à regulação, padrões, testes e certificações não criem obstáculos 
desnecessários ao comércio, permitindo aos Estados, contudo, que estes tomem medidas adequadas para a proteção da vida e da saúde de seres humanos, animais e vegetais. $\mathrm{O}$ referido Acordo aplica-se a características do produto e aos métodos de processo e produção que tenham efeito sobre as suas características e qualidade, tendo como intuito garantir padrões de qualidade e de segurança (BARROS; BURNQUIST; CUNHA FILHO; MIRANDA, 2004, p. 26).

Dessa forma, ambos os Acordos se aplicam ao comércio de bens e lidam com barreiras não-tarifárias ao comércio internacional. O Acordo SPS se aplica apenas às medidas específicas de proteção à vida ou à saúde humana, animal e vegetal de determinados riscos enumerados no Acordo. Já o Acordo TBT trata de medidas, referentes à regulação técnica, standards e processos de avaliação, adotadas por diversos objetivos, tais como segurança nacional e proteção da saúde ou do ambiente, não sendo esta uma lista exaustiva (GRUSZCZYNSKI, 2008, p. 16-17).

Além disso, disposições acerca da proteção à saúde e ao meio ambiente também se encontram no texto do GATT 1947/1994, especificamente no Artigo XX (b) e $(\mathrm{g})^{2}$. Os dispositivos permitem, como exceção geral ao GATT, a adoção de medidas necessárias à proteção da saúde e da vida das pessoas e dos animais e à preservação dos vegetais, assim como as relacionadas à preservação de recursos naturais esgotáveis, desde que não constituam meio de discriminação arbitrária ou injustificada, nem restrição disfarçada ao comércio.

Devem ser ressaltadas adiante as principais disposições constantes no Acordo SPS, uma vez que este norteia de forma direta a regulamentação da OMC sobre o tema da segurança alimentar ${ }^{3}$. O Acordo SPS objetiva encontrar o equilíbrio entre a garantia do direito de o Membro proteger seu mercado de produtos que possam ser prejudiciais à vida ou à saúde humana, animal ou vegetal, e a preservação dos princípios do livre comércio, que pode ser ameaçado diante da adoção de medidas disfarçadamente discriminatórias ou protecionistas (GUZMAN, 2007, p. 215).

Em primeiro lugar, observa-se que o Acordo define como medidas sanitárias ou fitossanitárias aquelas aplicadas para proteger a vida ou a saúde humana, animal ou vegetal de riscos resultantes de pragas, doenças ou organismos patogênicos ou portadores de doenças; e para proteger a vida ou a saúde humana ou animal de riscos provenientes de aditivos, contaminantes, toxinas ou organismos patogênicos em alimentos, bebidas ou ração animal (Artigo 1 do Anexo A). O Acordo SPS é, portanto, voltado à regulamentação 
Segurança alimentar e Organização Mundial do Comércio: análise crítica do acordo sobre a aplicação de medidas sanitárias e fitossanitárias

de medidas sanitárias e fitossanitárias (medidas SPS), quais sejam, aquelas que servem à proteção da vida ou saúde humana, animal ou vegetal.

As medidas SPS deverão ser adotadas pelos Estados de forma necessária a garantir a proteção almejada, baseada em princípios científicos, e não deverá ser mantida sem evidência científica suficiente para tanto, com exceção do determinado pelo Artigo 5.7 (Artigo 2.2). O citado artigo permite que o Estado mantenha uma medida SPS provisoriamente, no caso de provas científicas insuficientes, desde que estejam embasadas em informação pertinente disponível; nesse caso, o Estado deverá buscar a informação adicional necessária para uma avaliação mais objetiva do risco, e revisará a medida em um prazo razoável.

O Acordo determina, ainda, que as medidas SPS não devem acarretar discriminação arbitrária ou injustificada a outros Estados, nem devem configurar uma restrição velada ao comércio (Artigo 2.3). As medidas SPS serão consideradas necessárias à proteção da vida ou saúde humana, animal ou vegetal se estiverem em conformidade com normas, guias e recomendações internacionais, somente podendo acarretar um nível mais elevado de proteção se houver justificativa científica ou se for consequência do nível de proteção que o Estado determine ser apropriado (Artigo 3.2 e $3.3)$.

Ademais, os Estados devem garantir que as medidas SPS tomadas sejam baseadas em uma avaliação de risco, que deve levar em consideração as técnicas elaboradas pelas organizações internacionais competentes (Artigo 5.1). Através da avaliação realizada, os Estados devem determinar o nível adequado de proteção sanitária ou fitossanitária, e devem considerar fatores econômicos relevantes, tais como o dano potencial em termos de perda de produção ou de vendas no caso da entrada, estabelecimento e disseminação de uma peste ou doença, os custos de controle e de erradicação no território do Estado importador e a relação custo-benefício de enfoques alternativos para limitar os riscos (Artigo 5.3).

De uma maneira geral, portanto, pode-se afirmar que as medidas SPS devem estar baseadas em princípios científicos e não podem ser mantidas sem evidências científicas suficientes. Caso as medidas não estejam de acordo com os padrões internacionais, que promovem a harmonização das medidas SPS, o embasamento científico, alcançado pela avaliação de risco, é exigido. O processo de avaliação deve levar em consideração as evidências científicas disponíveis. No caso de sua insuficiência, pode ser adotada uma medida SPS, provisoriamente, com base nas informações 
pertinentes disponíveis; nesse caso, o Membro deverá buscar a obtenção de dados adicionais necessários para uma avaliação de risco mais objetiva (GRUSZCZYNSKI, 2006, p. 8-9).

Através da exposição das principais normas contidas no Acordo SPS, é possível verificar que o Acordo reconhece a soberania dos Estados para definir seus padrões de segurança alimentar, os quais podem ser, inclusive, de nível mais elevado que o dos padrões internacionais, designados, segundo o preâmbulo do Acordo, pelo Codex Alimentarius, pela OIE (World Organization for Animal Health) e pela IPPC (International Plant Protection Convention). Em contrapartida, o Acordo estabelece que as medidas SPS, mais protetivas que as registradas pelos standards internacionais, sejam adotadas ou mantidas com base em evidências científicas.

Dessa forma, as medidas, para serem consideradas em conformidade com o Acordo, não podem gerar uma discriminação injustificada ou arbitrária, nem disfarçadamente restritiva ao comércio. As medidas SPS são justificadas, portanto, diante de sua conformidade com os padrões internacionais representados pelo Codex Alimentarius, pela OIE e pela IPPC - caso no qual é presumida sua adequação às evidências científicas e, portanto, ao Acordo SPS - ou, se forem adotadas em um nível mais elevado de proteção, diante de provas científicas que demonstrem sua adequação e necessidade.

O Acordo SPS, portanto, reconhece o direito dos Membros da OMC de adotar medidas necessárias para a proteção da vida ou da saúde humana, animal ou vegetal, na observância dos princípios da não-discriminação e do tratamento nacional, de forma a não constituir restrição disfarçada ao comércio internacional. Além disso, os Membros devem aceitar como equivalentes as medidas SPS dos demais caso o Estado exportador demonstre equivalência. Em suma, o Acordo SPS demanda que os Membros garantam que as medidas SPS são adotadas somente na extensão necessária para proteger a saúde e o meio ambiente, são baseadas em princípios científicos e não são mantidas sem evidência científica suficiente (SANDS, 2003, p. 978).

Como se pode observar, o embasamento científico é fundamental para se justificar uma medida perante o Acordo SPS, uma vez que indica que o Estado não tem intenções protecionistas, mas sim o interesse legítimo de proteger sua população ou o meio ambiente. Sendo assim, caso o Estado possua standards de grau mais elevado que o dos internacionais, cabe a ele justificar suas medidas SPS, mediante evidências científicas, quando estas afetarem o comércio internacional. 
Segurança alimentar e Organização Mundial do Comércio: análise crítica do acordo sobre a aplicação de medidas sanitárias e fitossanitárias

O papel da ciência na solução de controvérsias tem-se mostrado um grande desafio para a OMC, que lidava principalmente com questões tarifárias. Nas controvérsias sob fundamento dos Acordos SPS e TBT, trata-se de riscos científicos e incertezas, os quais acabam por impactar o comércio internacional diante das regras impostas pelos Estados no sentido de proteção ambiental e da segurança alimentar, por exemplo (JAMES, 2000, p. 1-2).

Nota-se que, diferentemente das barreiras tarifárias, as quais podem ser reduzidas ou eliminadas mediante barganha entre chefes de Estados em negociações comerciais, as barreiras não-tarifárias, como a barreira imposta para proteção da segurança alimentar, representam obstáculos mais complexos de lidar no âmbito do comércio internacional, uma vez que se trata de regulações que visam a proteger questões relacionadas à saúde, à segurança e ao bem-estar dos consumidores e do meio ambiente. A grande dificuldade que se impõe diante das barreiras regulatórias é de se mensurar até que ponto esses padrões de proteção são adotados pelo interesse público ou se constituem em novas formas de discriminação no comércio internacional (KOTZIAS; THORSTENSEN, 2015, p. 82).

\section{A INTERPRETAÇÃO DO ACORDO SPS SEGUNDO O ÓRGÃO DE SOLUÇÃO DE CONTROVÉRSIAS DA OMC: A DISPUTA EC-BIOTECH}

Sendo assim, faz-se necessário compreender o entendimento da OMC acerca das situações em que uma medida sanitária ou fitossanitária, no caso deste estudo, uma medida relacionada à segurança alimentar, deve ser interpretada como necessária e proporcional à proteção de interesses públicos ou como uma restrição infundada ou arbitrária ao comércio internacional. Tal análise será realizada em seguida a partir da interpretação conferida às principais normas previstas no Acordo SPS mediante a uma das controvérsias de maior destaque levadas ao Órgão de Solução de Controvérsias (OSC) da Organização, cujo objeto de disputa se refere a uma medida abarcada pelo Acordo SPS: o caso EC-Biotech Products.

A questão considerada controversa diz respeito à reclamação da Argentina, do Canadá e dos Estados Unidos, formulada em 2003, no tocante a duas situações: a aplicação pela União Europeia do seu regime de aprovação de produtos geneticamente modificados e a adoção de medidas, pela UE, no sentido de proibir ou restringir o comércio desses produtos ${ }^{4}$. 
A legislação aplicável da União Europeia demandava que fosse realizada uma avaliação, de acordo com cada caso, dos riscos potenciais que os produtos transgênicos poderiam eventualmente representar à saúde e ao meio ambiente. Além disso, segundo a legislação europeia, havia a possibilidade de os Estados-membros assumirem medidas de salvaguarda em relação aos produtos geneticamente modificados que tivessem obtido aprovação para o comércio na UE. Sendo assim, poderia haver, por parte de um Estadomembro, a restrição ou proibição provisória da utilização e/ou venda de um produto transgênico aprovado em seu próprio território na hipótese de existência de razões válidas para considerar, com base em informação nova ou conhecimento científico, que aquele determinado produto representaria um risco para a saúde humana ou para o ambiente. A adoção de medidas de salvaguarda deveria ser comunicada aos outros Estados-membros para que se chegasse a uma decisão em nível comunitário ${ }^{5}$.

Houve, portanto, o questionamento quanto ao fato de a legislação incidente sobre a União Europeia permitir que os Estados-membros pudessem, a título de salvaguarda, adotar medidas unilaterais de restrição da comercialização de produtos geneticamente modificados, embora esta tenha obtido uma aprovação prévia na UE. Para tanto, bastaria a alegação por parte de um Estado integrante de que, diante da superveniência de conhecimento científico novo ou adicional, seria possível acreditar que o produto ensejasse risco à saúde ou ao meio ambiente.

De acordo com os reclamantes, a União Europeia teria instaurado uma moratória de fato aos organismos geneticamente modificados (OGMs), violando os seus deveres como membro da $\mathrm{OMC}$, ainda que a pretexto de proteger a saúde e o meio ambiente. Ademais, os reclamantes sustentaram que os produtos transgênicos teriam trazido vários benefícios quanto a esses aspectos, tendo sido sua segurança constatada, inclusive, pela FAO e pela $\mathrm{OMS}^{6}$.

$\mathrm{Na}$ defesa da União Europeia, esta refutou a alegação de que havia estabelecido uma moratória de fato ou incorrido em atraso injustificado. Afirmou que, entre 1998 e 2001, período em houve o suposto atraso indevido, havia uma intensa controvérsia acerca dos OGMs, o que, inclusive, levou à revisão da metodologia de avaliação de risco desses produtos ${ }^{7}$. Aduziu, ainda, que a matéria não poderia ser abordada somente sob o prisma das normas da OMC, mas interpretada sob a ótica das demais normas de Direito Internacional Público, destacando as contidas no Protocolo de Cartagena sobre Biossegurança e na Convenção sobre Diversidade Biológica ${ }^{8}$. 
Segurança alimentar e Organização Mundial do Comércio: análise crítica do acordo sobre a aplicação de medidas sanitárias e fitossanitárias

Em relação às consequências dos organismos geneticamente modificados à saúde e ao ambiente, a UE ressaltou a insipiência das pesquisas científicas, o que ensejou a adoção de uma postura prudente tanto da UE quanto de outros países no tocante à avaliação do impacto ambiental e sanitário de novas tecnologias, tais como os OGMs. Tal postura estaria baseada no princípio da precaução, norma consuetudinária ou, pelo menos, princípio geral do Direito Internacional, previsto, por exemplo, na Declaração do Rio sobre Meio Ambiente e Desenvolvimento e no Protocolo de Cartagena sobre Biossegurança9 ${ }^{9}$. Destacou o Artigo 5.7 do Acordo SPS (Acordo sobre a Aplicação de Medidas Sanitárias e Fitossanitárias), no âmbito normativo da OMC, que permitiria a utilização provisória de medidas sanitárias e fitossanitárias no caso de evidência científica relevante insuficiente. Afirma, nesse sentido, que o conceito de suficiência, presente no mencionado Artigo, é relativo, podendo cada Membro estabelecer seus próprios níveis adequados de proteção sanitária, que podem ser mais elevados que os padrões internacionais existentes, a depender da legislação de cada um $^{10}$.

No relatório final, o Panel confirmou a relevância de acordos internacionais que não as normas da OMC, porém apenas aqueles que estabelecessem relação jurídica entre as partes, o que não ocorreu no caso em apreço ${ }^{11}$.

O exame da controvérsia foi dividido em três tópicos: 1) a moratória de fato da União Europeia; 2) as medidas da UE contra o processo de aprovação de produtos específicos; 3 ) as medidas de salvaguarda adotadas pelos países-membros da UE afetando a comercialização desses produtos ${ }^{12}$. Antes da análise desses pontos, abordou-se a aplicabilidade do Acordo SPS à hipótese em exame, concluindo-se que as normas europeias especificadas pelos reclamantes - as Diretivas 90/220 e 2001/18, bem como o Regulamento 258/97 - integram medidas sanitárias e fitossanitárias albergadas pelo referido Acordo ${ }^{13}$.

No que tange à moratória de fato aos organismos geneticamente modificados, pela União Europeia, entre junho de 1999 e agosto de 2003, o Panel entendeu pela sua configuração. O relatório evidenciou que o denominado Grupo dos Cinco, composto por Dinamarca, Grécia, França, Itália e Luxemburgo, obstou, propositalmente, os processos de aprovação de OGMs, tendo a Comissão Europeia cooperado com tal conduta ao se omitir diante desse fato, que era de seu conhecimento. Sendo assim, o Panel entendeu que, não tendo a Comissão usado seus poderes para completar os processos de aprovação, e tendo plena ciência das dificuldades impostas a esses procedimentos por uma minoria, ela foi conivente com o comportamento adotado pelo Grupo dos Cinco, o que se permitiu 
dizer que houve a alegada moratória de fato aos produtos transgênicos na União Europeia no citado período. A moratória aplicada denomina-se de fato porque não foi admitida mediante norma ou decisão formal da $\mathrm{UE}^{14}$.

Estando presente, de acordo com as conclusões obtidas pelo Panel, o atraso no processo de avaliação de risco para a aprovação dos OGMs, ainda se demonstrou que o atraso foi injustificável, haja vista a necessidade de adaptação normativa não constituir motivo para a moratória adotada, bem como a legislação aplicável à União Europeia não fornecer um embasamento legal apto a impor medidas adicionais para a autorização dos produtos geneticamente modificados. Acerca do princípio da precaução como alegada justificativa para o atraso no processo em questão, o Panel afirma que, em tese, não há incompatibilidade entre uma abordagem precaucionária e o Acordo SPS, desde que sujeita aos limites da razoabilidade. A complexidade e a incerteza científica, embora demandassem a adoção de medidas sanitárias e fitossanitárias recomendáveis, não impediriam a conclusão do processo de avaliação de risco sob o ponto de vista da prudência e da precaução ${ }^{15}$.

No que diz respeito às medidas de salvaguarda, estas foram nove adotadas por países-membros - Áustria, Bélgica, França, Alemanha, Itália e Luxemburgo - em relação à comercialização de OGMs já autorizada pela União Europeia. O Panel entendeu que, como a análise desses produtos já havia sido submetida aos comitês científicos da UE, que, avaliando os eventuais riscos à saúde e ao meio ambiente, aprovaram a comercialização dos produtos transgênicos, não haveria justificativa para que permanecessem as barreiras comerciais levantadas pelos citados países-membros ${ }^{16}$.

Desse modo, embora a União Europeia alegasse que as medidas adotadas correspondiam ao seu direito de estabelecer padrões mais elevados de proteção e que o atraso decorrente do processo de avaliação de risco dos OGMs ocorreu em razão da incipiência científica e complexidade em torno do tema, o Panel concluiu que as medidas não estavam justificadas pelo Acordo SPS. Conforme já explicitado neste estudo, o direito do Estado de fixar standards mais rígidos de proteção sanitária ou fitossanitária, previsto no Artigo 3.1, não é absoluto, logo não isenta o Membro de cumprir os dispositivos contidos no Acordo, como se extrai do Artigo 3.3.

Ademais, a inexistência de evidência científica suficiente acerca dos organismos transgênicos não justifica a adoção das medidas no caso em tela. Primeiramente, não se legitima em virtude de os OGMs submetidos às medidas de salvaguarda já terem sido aprovados para a comercialização pelos comitês científicos da 
Segurança alimentar e Organização Mundial do Comércio: análise crítica do acordo sobre a aplicação de medidas sanitárias e fitossanitárias

União Europeia, isto é, já havia sido constatado, através de embasamento científico, sua segurança para a população.

Em segundo lugar, não haveria que se falar na aplicação do Artigo 5.7 à controvérsia, uma vez que o dispositivo demanda a ausência de evidência científica suficiente sobre o risco do produto para que seja justificável a adoção de uma medida SPS provisoriamente. Aliás, o Artigo não permite que tal ausência seja fundamento autônomo para impedir a importação de determinado produto, mas exige que seja realizada uma avaliação de risco e que a medida seja revista em tempo razoável.

No caso da União Europeia, procedeu-se aos processos de avaliação, porém estes levaram muito tempo sem que pudesse ser constatada a segurança dos organismos transgênicos submetidos, e a União Europeia não apresentou qualquer justificativa válida para tanto. O princípio da precaução, ainda que esteja previsto, de certa forma, no Acordo SPS, especialmente no Artigo 5.7, não legitima, por si só, a adoção de medidas protetivas não justificadas pelo Acordo.

Percebe-se, portanto, mediante o estudo do caso EC-Biotech, que o Acordo SPS é estruturado pelo embasamento científico, que, especialmente por meio dos padrões internacionais, garante a legitimidade de uma medida sanitária ou fitossanitária. A avaliação dos riscos também constitui uma parte fundamental do Acordo SPS, uma vez que demonstra a intenção do Membro de atestar a segurança de um produto através de métodos científicos. Como os Acordos da OMC são permeados pelos princípios gerais do GATT, a não-discriminação é uma norma essencial também no Acordo SPS, visto que as medidas SPS não podem ser utilizadas com intuito discriminatório ou protecionista.

\section{CAMINHOS PARA A FORMAÇÃO DE UMA POLÍTICA REGULATÓRIA EM CONFORMIDADE COM O ACORDO SPS}

Após a análise dos dispositivos constantes no Acordo SPS, assim como do exame do caso EC-Biotech, é possível reunir alguns elementos aptos a demonstrar quando uma medida sanitária ou fitossanitária está conforme ao Acordo, segundo a interpretação do OSC (KENNEDY; SOUTHWICK, 2002, p. 558-573).

O primeiro elemento é o impacto gerado pela medida, isto é, a medida não afeta negativamente a comercialização de determinado produto sem se aplicar da mesma maneira para um produto nacional ou de outro país que não o prejudicado. $\mathrm{Na}$ controvérsia Japan-Agricultural Products II, por exemplo, a medida que demandava um 
processo específico de quarentena para os produtos importados não se aplicava aos produtos domésticos concorrentes, o que denota um intuito protecionista.

O segundo elemento diz respeito à conformidade com os padrões internacionais. Como já explicitado anteriormente, os Membros deverão basear suas medidas SPS em normas, guias e recomendações internacionais (Artigo 3.1), exceto se o Acordo SPS dispuser o contrário, em especial o Artigo 3.3, que permite a adoção pelos Membros de medidas com nível mais elevado de proteção que o fornecido pelos padrões internacionais, desde que haja justificação científica ou que decorra de um grau de proteção considerado apropriado e respeite os dispositivos do Acordo.

No caso EC-Hormones, por exemplo, a maioria dos hormônios objetos da medida de restrição discutida era permitida pela Comissão do Codex Alimentarius. Sendo assim, a medida adotada pela União Europeia violou o Artigo 3.1, haja vista ser mais restrita que o demandado pelos standards internacionais e não haver sido justificada nos parâmetros do Artigo 3.3.

O terceiro elemento trata da presença de justificativas para a adoção de medidas SPS. A principal justificativa de uma medida SPS é o embasamento científico (Artigo 2.2) através da avaliação de riscos (Artigo 5.1). Outra justificativa é o gerenciamento de riscos, uma vez que o Acordo demanda que a medida não seja mais restritiva ao comércio que o necessário para atingir o nível adequado de proteção pretendido pelo Membro (Artigo 5.6), bem como que não enseje discriminação arbitrária ou infundada (Artigo 5.5).

Compreendidos os elementos que devem compor uma política de regulação voltada à proteção da segurança alimentar, é importante que se destaque a relação entre os dispositivos do Acordo SPS a fim de que se possa entender plenamente as obrigações dos Estados conforme a interpretação da OMC.

Na disputa EC-Biotech, o Panel estabeleceu que o Artigo 3.3, que possibilita a adoção de medidas SPS com nível mais elevado de proteção que os padrões internacionais, constitui um direito autônomo ao disposto no Artigo 3.1, e não uma exceção à obrigação geral por ele imposta. Essa relação de autonomia ocorre quando um dispositivo (no caso, o Artigo 3.3) permite, em determinadas circunstâncias, um comportamento que, em outra situação, seria inconsistente com o outro dispositivo (no caso, com a obrigação contida no Artigo 3.1 de basear as medidas SPS em padrões internacionais); quando um dos dispositivos se refere ao outro dispositivo (o Artigo 3.1 refere-se ao Artigo 3.3); e quando um dos dispositivos sugere que a obrigação instituída 
Segurança alimentar e Organização Mundial do Comércio: análise crítica do acordo sobre a aplicação de medidas sanitárias e fitossanitárias

(conformidade das medidas SPS com padrões internacionais) não se aplica às medidas abrangidas pelo outro dispositivo (o Artigo 3.1 afirma que o disposto no Acordo pode excepcionar essa obrigação, especialmente o prescrito no Artigo 3.3) ${ }^{17}$.

Isso significa que o Artigo 3.1 exclui do seu âmbito de aplicação as situações regulamentadas pelo Artigo 3.3. Dessa maneira, a eventual desconformidade de uma medida SPS com o Artigo 3.3 não gera, necessariamente, a inconsistência com o Artigo 3.1, já que se trata de direitos distintos com esferas de incidência diferentes. Além disso, a importância de os Artigos 3.1 e 3.3 serem considerados direitos autônomos reside no fato de o OSC entender que, nesse caso, o ônus da prova quanto à inconsistência da medida SPS com o dispositivo recai, em uma disputa submetida ao OSC, no Estado reclamante, e não no Estado reclamado ${ }^{18}$.

Uma relação similar é encontrada, no caso EC-Biotech, no que tange aos Artigos 2.2 e 5.7, sendo este último o que permite a adoção provisória de uma medida SPS com base na informação pertinente disponível nas hipóteses de evidência científica insuficiente. O referido Artigo não é considerado uma exceção à obrigação geral prevista no Artigo 2.2 ${ }^{19}$. Portanto, desse entendimento decorre que o ônus da prova no que concerne a incompatibilidade da medida SPS incumbe ao Estado reclamante no OSC ${ }^{20}$.

O mesmo teste aplicado aos Artigos 3.1 e 3.3 pode ser admitido no que diz respeito aos Artigos 2.2 e 5.7 para confirmar que existe entre eles uma relação de autonomia, e não de regra/exceção. Um dispositivo (no caso, o Artigo 5.7) é considerado um direito, e não uma exceção, quando permite, em determinadas circunstâncias, um comportamento que, em outra situação, seria inconsistente com o outro dispositivo (no caso, com a obrigação contida no Artigo 2.2 de não manter uma medida SPS sem evidência científica suficiente); quando um dos dispositivos se refere ao outro dispositivo (o Artigo 2.2 refere-se ao Artigo 5.7); e quando um dos dispositivos sugere que a obrigação imposta (não manutenção de uma medida SPS sem evidência científica suficiente) não se aplica às medidas abrangidas pelo outro dispositivo (o Artigo 2.2 coloca como exceção a essa obrigação o disposto no Artigo 5.7 $)^{21}$.

Embora seja possível estabelecer que não existe o binômio regra/exceção nas relações entre os Artigos 3.1 e 3.3 e os Artigos 2.2 e 5.7, essas relações não são idênticas. Nota-se que, na hipótese dos Artigos 3.1 e 3.3, o Estado pode optar entre adotar uma medida SPS com base nos padrões internacionais (Artigo 3.1) ou, alternativamente, justificar cientificamente uma medida SPS a fim de alcançar um nível de proteção mais elevado (Artigo 3.3). No caso dos Artigos 2.2 e 5.7, se não houver evidência científica 
suficiente (o que permite a aplicação do Artigo 5.7), a escolha de não manter uma medida SPS sem justificação científica suficiente (Artigo 2.2) torna-se impossívelel22.

O Artigo 5.7 contém quatro requisitos, de acordo com o OSC, quais sejam, a medida deve se fazer necessária ante uma situação de insuficiência de evidência científica relevante; a medida deve ser adotada com base em informação pertinente disponível; o Membro deve buscar a obtenção da informação complementar necessária para uma análise de risco mais objetiva; e o Membro deve rever a medida em um prazo razoável ${ }^{23}$. O disposto no Artigo 2.2 não regula as situações abarcadas pelo Artigo 5.7, logo não se trata de uma relação regra/exceção. Apenas quando não cumprido um desses requisitos, passa a ser aplicável a obrigação instituída no Artigo 2.2, que demanda não ser mantida uma medida SPS sem evidência científica suficiente. Diante dessa interpretação, o OSC caracteriza o Artigo 5.7 como uma exceção qualificada (qualified exemption) do Artigo $2.2^{24}$. Ademais, o OSC entende que, caso um Estado alegue a inconsistência de uma medida SPS com o Artigo 2.2, cabe a ele, e não ao Estado reclamado, provar a incompatibilidade com pelo menos um dos requisitos do Artigo $5.7^{25}$.

Do ponto de vista deste artigo, no entanto, a automática aplicação do Artigo 2.2 quando houver o descumprimento de um dos requisitos do Artigo 5.7 padece de uma incoerência lógica com os demais argumentos expostos no relatório do Panel na disputa EC-Biotech. No caso de uma medida SPS descumprir um dos requisitos previstos no Artigo 5.7, por exemplo, a revisão da medida em prazo razoável, segundo o OSC, a medida violaria o Artigo 5.7 e, automaticamente, estaria inconsistente com o Artigo 2.2. Entretanto, nessa hipótese, há insuficiência de evidência científica e, ainda assim, o OSC entende que a medida descumpriu o Artigo 2.2, que exige a não manutenção de medida SPS sem evidência científica suficiente. Percebe-se, portanto, que a interpretação de que o descumprimento do Artigo 5.7 leva à inconsistência imediata com o Artigo 2.2 carece de sentido, uma vez que permite que uma medida SPS, simplesmente por não cumprir um dos requisitos do Artigo 5.7, seja incompatível com o Artigo 2.2 mesmo que tenha sido adotada em razão de evidência científica insuficiente (GRUSZCZYNSKI: 2009; p. 66).

Tal incongruência foi levantada, ainda que não expressamente, na disputa Japan - Agricultural Products II, em que o Japão sustenta que a frase contida no Artigo 2.2, "exceto o disposto no parágrafo 7 do Artigo 5", apenas se aplica ao primeiro requisito do Artigo 5.7, qual seja, o de insuficiência científica. Dessa forma, segundo o argumento do Japão, apenas na hipótese de ser descumprido o requisito de insuficiência científica, e 
Segurança alimentar e Organização Mundial do Comércio: análise crítica do acordo sobre a aplicação de medidas sanitárias e fitossanitárias

não os demais requisitos presentes no Artigo 5.7, restaria violado o Artigo 2.2, que demanda que a medida SPS não seja mantida sem evidência científica suficiente ${ }^{26}$.

Ao se analisar a interação dos principais dispositivos do Acordo SPS, percebe-se que, diante do Acordo, são três os caminhos possíveis para a estruturação de uma política regulatória estatal direcionada a um nível desejado de proteção da segurança alimentar do Estado sem oferecer barreiras protecionistas ao comércio internacional (GRUSZCZYNSKI, 2009, p. 66-67).

O primeiro caminho é a adequação da política de regulação aos padrões internacionais dispostos pelo Codex Alimentarius, pela OIE e pela IPPC, conforme estabelecido no Artigo 3.1 do Acordo. Segundo o citado dispositivo, como já abordado, as medidas SPS cujo nível de proteção equivale ao nível proposto pelos padrões internacionais presumem-se consistentes com o Acordo SPS, haja vista esses padrões já contarem com o embasamento científico necessário para justificar a adoção de tais medidas.

O segundo caminho é o permitido pelo Artigo 3.3 do Acordo SPS, que prevê a possibilidade de o Estado, caso deseje um nível mais elevado de proteção sanitária ou fitossanitária, adotar medidas SPS mais rigorosas que as dispostas nos padrões internacionais. Para tanto, exige-se que o Estado baseie sua medida em evidência científica e avaliação de riscos.

É necessário, então, que o Estado identifique o risco, por meio de embasamento científico, e, a partir daí, determine o grau de proteção almejado, o que deve estabelecer, por consequência, uma relação de racionalidade entre a medida SPS e o resultado da avaliação de riscos. Deve-se ressaltar que esse risco não pode ser hipotético, uma vez que a ciência não é capaz de garantir com toda certeza a segurança de produtos, mas sim um risco verificável através de uma avaliação (GUZMAN, 20042005, p. 9-10).

Finalmente, o terceiro caminho se aplica às situações regulamentadas pelo Artigo 5.7 do Acordo SPS, em que a evidência científica é insuficiente para se obter uma avaliação dos riscos, logo o Estado não está obrigado a cumprir os requisitos de cientificidade exigidos no Artigo 2.2. Nesses casos, o Estado poderá adotar, provisoriamente, a medida SPS de acordo com a informação pertinente disponível e cumprir os demais requisitos presentes no Artigo 5.7, quais sejam, buscar obter a informação adicional necessária para uma avaliação mais objetiva do risco e revisar a medida em um prazo razoável. 
Portanto, a melhor forma de se estabelecer uma relação harmônica entre os citados dispositivos é considerá-los direitos autônomos conferidos aos Estados. Desse modo, dentre os três caminhos existentes, a eventual inconsistência de uma medida SPS com os dispositivos aplicáveis a cada opção não permite que a medida passe a ser regulada por dispositivos não incidentes sobre aquela opção. Por conseguinte, sendo os dispositivos concebidos como direitos autônomos, a aplicação de um dispositivo exclui da sua área de incidência medidas suscetíveis de regulamentação por outros dispositivos independentes (GRUSZCZYNSKI, 2009, p. 67).

\section{CONCLUSÃO}

O estudo do Acordo SPS é imprescindível para que se possa compreender o limite da razoabilidade de uma política regulatória voltada à garantia da segurança alimentar. Por um lado, ao Estado cabe assegurar as condições necessárias para o bemestar da população, logo é essencial que sejam adotadas medidas aptas à proteção da vida e da saúde humana e do meio ambiente. Por outro lado, tais prerrogativas do Estado não podem ser empregadas como barreiras comerciais protecionistas, por isso a OMC aponta as premissas básicas de uma legítima medida SPS.

De forma geral, as medidas SPS devem ser justificadas em princípios científicos e não podem ser mantidas sem evidências científicas suficientes (Artigo 2.2). Caso as medidas não estejam em conformidade com os padrões internacionais (Artigo 3.1), que promovem a harmonização das medidas SPS, o embasamento científico, alcançado pela avaliação de risco, é requerido (Artigo 3.3). O processo de avaliação deve levar em consideração as evidências científicas disponíveis (Artigo 5.2). No caso de sua insuficiência, pode ser adotada, provisoriamente, uma medida SPS com base nas informações pertinentes disponíveis; nessa hipótese, o Membro deverá buscar a obtenção de dados adicionais necessários para uma avaliação de risco mais objetiva (Artigo 5.7).

Os Artigos 2.2, 3.1, 3.3 e 5.7 do Acordo SPS constituem direitos autônomos concedidos aos Estados e direcionam sua política regulatória de segurança alimentar em três caminhos: a adequação das medidas aos padrões internacionais; a adoção de medidas, justificadas cientificamente, que elevem o nível de proteção; e a adoção provisória de medidas, em razão de insuficiência científica, com base na informação pertinente disponível. 
Segurança alimentar e Organização Mundial do Comércio: análise crítica do acordo sobre a aplicação de medidas sanitárias e fitossanitárias

Sendo cada um desses dispositivos constitutivos de um direito autônomo, a aplicação de um deles exclui a incidência dos outros, logo a inconsistência de uma medida com um deles não leva, necessariamente, ao descumprimento dos demais. Além disso, o reconhecimento dos dispositivos citados como direitos permite, em uma controvérsia submetida ao OSC, conferir o ônus da prova quanto à inconsistência da medida com o Acordo SPS ao Estado reclamante.

\section{REFERÊNCIAS}

BARROS, G.S.A.C.; BURNQUIST, H.L.; CUNHA FILHO, J.H.; MIRANDA, S.H.G. Normas sanitárias e fitossanitárias: proteção ou protecionismo. Informações Econômicas, v. 34, n. 2, p. 25-35, 2004.

ETCHELAR, Emilie; MALJEAN-DUBOIS, Sandrine. World Trade and International Normalisation: Codex Alimentarius. WTO Obligations and Opportunities: Challenges of Implementation, 2007, p. 121-154.

GRUSZCZYNSKI, Lukasz. Insufficiency of Scientific Evidence under Article 5.7 of SPS Agreement: Some Remarks on the Panel Report in the EC - Biotech Products Case. University of Ottawa Law \& Technology Journal, Vol. 6, No. 1-2, 2009, p. 53-78.

GRUSZCZYNSKI, Lukasz. The Role of Science in Risk Regulation under the SPS Agreement. EUI Law Working Paper, No. 2006/03, February 2006.

GRUSZCZYNSKI, Lukasz. The SPS Agreement within the Framework of WTO Law: The Rough Guide to the Agreement's Applicability. European University Institute, October, 2008.

GUZMAN, Andrew. Dispute Resolution in SPS Cases. Ten Years of WTO Dispute Settlement. Ed. Horovitz, Moulis, and Steger. London: International Bar Association, 2007, 215-233.

GUZMAN, Andrew T. Food Fears: Health and Safety at the WTO. 45 Va. J. Int'l L. 1, 2004-2005, p. 1-39

JAMES, Sallie. An Economic Analysis of Food Safety Issues Following the SPS Agreement: Lessons from the Hormones Dispute. CIES Working Paper No. 5, February, 2000.

KENNEDY, Daniel L. M.; SOUTHWICK, James D., (Eds.). The political economy of international trade law: Essays in honor of Robert L. Hudec. Cambridge: Cambridge University Press, 2002.

KOTZIAS, Fernanda; THORSTENSEN, Vera. Barreiras regulatórias: um novo desafio para a governança da OMC. Política Externa (USP), 2015, v. 24, p. 81-92. 
LIVERMORE, Michael A. Authority and Legitimacy in Global Governance: Deliberation, Institutional Differentiation, and the Codex Alimentarius. New York University Law Review, 2006, Vol. 81, p. 766-801.

MATTHEW, Richard A.; MCDONALD, Bryan. Food Security in a Global Age: Addressing Challenges from Malnutrition, Food Safety and Environmental Change. APSA 2009 Toronto Meeting Paper.

PINSTRUP-ANDERSEN, P. Food security: definition and measurement. Food Security, 2009, 1:5-7.

RANDELL, A. W.; WHITEHEAD, A. J. Codex Alimentarius: food quality and safety standards for international trade. Rev. sci. tech. Off. int. Epiz., 1997, 16 (2), p. 313-321

SANDS, Philippe. Principles of International Environmental Law. Cambridge: Cambridge University Press, 2003.

TOMA-BIANOV, Ana Maria. Codex Alimentarius Commission and the World Trade Organization Legal Order. Bulletin of the Transilvania University of Braşov, Series VII, Vol. 6 (55) No. 1-2013, p. 191-196.

WORLD TRADE ORGANIZATION. European Communities - Measures affecting the approval and marketing of biotech products: reports of the Panel. WT/DS291/R, WT/DS292/R, WT/DS293/R (29 Sep. 2006).

WORLD TRADE ORGANIZATION. Japan - Measures affecting agricultural products AB-1998-8: report of the Appellate Body. WT/DS76/AB/R (22 Feb. 1999).

Artigo recebido 30 de dezembro de 2016 e aceito em 15 de janeiro de 2017

\footnotetext{
${ }^{1}$ Informação disponível em: <http://www.fao.org/fao-who-codexalimentarius/about-codex/en/>. Acesso em: 29 set. 2016.

2 Texto do GATT disponível em: 〈https://www.wto.org/english/docs_e/legal_e/gatt47_e.pdf>. Acesso em 26 set. 2016.

${ }^{3}$ Texto em português do Acordo sobre a Aplicação de Medidas Sanitárias e Fitossanitárias disponível 
Segurança alimentar e Organização Mundial do Comércio: análise crítica do acordo sobre a aplicação de medidas sanitárias e fitossanitárias

$<$ http://www.agricultura.gov.br/arq_editor/file/vegetal/dsv/Acordo\%20SPS\%20em\%20Port.pdf >. Acesso em: 11 set. 2016.

4 Informação disponível na página oficial da Organização Mundial do Comércio: <https://www.wto.org/english/tratop_e/dispu_e/cases_e/ds291_e.htm>. Acesso em: 25 out. 2016.

${ }^{5}$ WORLD TRADE ORGANIZATION. European Communities - Measures affecting the approval and marketing of biotech products: reports of the Panel. WT/DS291/R, WT/DS292/R, WT/DS293/R (29 Sep. 2006), par. 2.1-2.5.

${ }^{6}$ WORLD TRADE ORGANIZATION. European Communities - Measures affecting the approval and marketing of biotech products: reports of the Panel. WT/DS291/R, WT/DS292/R, WT/DS293/R (29 Sep. 2006), par. 4.136-4.142.

${ }^{7}$ WORLD TRADE ORGANIZATION. European Communities - Measures affecting the approval and marketing of biotech products: reports of the Panel. WT/DS291/R, WT/DS292/R, WT/DS293/R (29 Sep. 2006), par. 4.339-4.340.

${ }^{8}$ WORLD TRADE ORGANIZATION. European Communities - Measures affecting the approval and marketing of biotech products: reports of the Panel. WT/DS291/R, WT/DS292/R, WT/DS293/R (29 Sep. 2006), par. 7.49-7.55.

9 WORLD TRADE ORGANIZATION. European Communities - Measures affecting the approval and marketing of biotech products: reports of the Panel. WT/DS291/R, WT/DS292/R, WT/DS293/R (29 Sep. 2006), par. 4.523-4.524.

${ }^{10}$ WORLD TRADE ORGANIZATION. European Communities - Measures affecting the approval and marketing of biotech products: reports of the Panel. WT/DS291/R, WT/DS292/R, WT/DS293/R (29 Sep. 2006), par. 4.761.

${ }^{11}$ WORLD TRADE ORGANIZATION. European Communities - Measures affecting the approval and marketing of biotech products: reports of the Panel. WT/DS291/R, WT/DS292/R, WT/DS293/R (29 Sep. 2006), par. 7.71-7.72.

${ }^{12}$ WORLD TRADE ORGANIZATION. European Communities - Measures affecting the approval and marketing of biotech products: reports of the Panel. WT/DS291/R, WT/DS292/R, WT/DS293/R (29 Sep. 2006), par. 8.12.

${ }^{13}$ WORLD TRADE ORGANIZATION. European Communities - Measures affecting the approval and marketing of biotech products: reports of the Panel. WT/DS291/R, WT/DS292/R, WT/DS293/R (29 Sep. 2006), par. 7.436.

14 WORLD TRADE ORGANIZATION. European Communities - Measures affecting the approval and marketing of biotech products: reports of the Panel. WT/DS291/R, WT/DS292/R, WT/DS293/R (29 Sep. 2006), par. 7.1272-7.1274.

15 WORLD TRADE ORGANIZATION. European Communities - Measures affecting the approval and marketing of biotech products: reports of the Panel. WT/DS291/R, WT/DS292/R, WT/DS293/R (29 Sep. 2006), par. 7.1522-7.1529.

${ }^{16}$ WORLD TRADE ORGANIZATION. European Communities - Measures affecting the approval and marketing of biotech products: reports of the Panel. WT/DS291/R, WT/DS292/R, WT/DS293/R (29 Sep. 2006), par. 8.8-8.9. 
17 Teste desenvolvido na resolução da controvérsia EC-Tariff Preferences. WORLD TRADE ORGANIZATION. European Communities - Measures affecting the approval and marketing of biotech products: reports of the Panel. WT/DS291/R, WT/DS292/R, WT/DS293/R (29 Sep. 2006), par. 7.2965-7.2966.

18 WORLD TRADE ORGANIZATION. European Communities - Measures affecting the approval and marketing of biotech products: reports of the Panel. WT/DS291/R, WT/DS292/R, WT/DS293/R (29 Sep. 2006), par. 7.2963.

${ }^{19}$ WORLD TRADE ORGANIZATION. European Communities - Measures affecting the approval and marketing of biotech products: reports of the Panel. WT/DS291/R, WT/DS292/R, WT/DS293/R (29 Sep. 2006), par. 7.2969.

${ }^{20}$ WORLD TRADE ORGANIZATION. European Communities - Measures affecting the approval and marketing of biotech products: reports of the Panel. WT/DS291/R, WT/DS292/R, WT/DS293/R (29 Sep. 2006), par. 7.2976.

${ }^{21}$ WORLD TRADE ORGANIZATION. European Communities - Measures affecting the approval and marketing of biotech products: reports of the Panel. WT/DS291/R, WT/DS292/R, WT/DS293/R (29 Sep. 2006), par. 7.2968.

${ }^{22}$ WORLD TRADE ORGANIZATION. European Communities - Measures affecting the approval and marketing of biotech products: reports of the Panel. WT/DS291/R, WT/DS292/R, WT/DS293/R (29 Sep. 2006), par. 7.2983.

${ }^{23}$ WORLD TRADE ORGANIZATION. European Communities - Measures affecting the approval and marketing of biotech products: reports of the Panel. WT/DS291/R, WT/DS292/R, WT/DS293/R (29 Sep. 2006), par. 7.2972.

${ }^{24}$ WORLD TRADE ORGANIZATION. European Communities - Measures affecting the approval and marketing of biotech products: reports of the Panel. WT/DS291/R, WT/DS292/R, WT/DS293/R (29 Sep. 2006), par. 7.2972-7.2973.

${ }^{25}$ WORLD TRADE ORGANIZATION. European Communities - Measures affecting the approval and marketing of biotech products: reports of the Panel. WT/DS291/R, WT/DS292/R, WT/DS293/R (29 Sep. 2006), par. 7.2976.

${ }^{26}$ WORLD TRADE ORGANIZATION. Japan - Measures Affecting Agricultural Products AB-1998-8: report of the Appellate Body. WT/DS76/AB/R (22 Feb. 1999), par. 11; 90. 